\title{
Inviscid Faraday waves in a brimful circular cylinder
}

\author{
R. Kidambi \\ Computational \& Theoretical Fluid Dynamics Division, \\ National Aerospace Laboratories, Bangalore 560017, \\ Council of Scientific \& Industrial Research, India. \\ Telefax: +91 8025220952 \\ email: kidambi@ctfd.cmmacs.ernet.in
}

(Received 20 March 2013)

We study inviscid Faraday waves in a brimful circular cylinder with pinned contact line. The pinning leads to a coupling of the Bessel modes and leads to an infinite system of coupled Mathieu equations. For large Bond numbers, even though the stability diagrams and the subharmonic and harmonic resonances for the free and pinned contact lines are similar, the free surface shapes can be quite different. With decreasing Bond number, not only are the harmonic and subharmonic resonances very different from the free contact line case but also interesting changes in the stability diagram occur with the appearance of combination resonance tongues. Points on these tongue boundaries correspond to almost periodic states. These do not seem to have been reported in the literature.

\section{Introduction}

Faraday waves are surface capillary-gravity waves generated when a body of fluid with a free surface is subject to an oscillatory acceleration, of frequency $\Omega$ and amplitude $\epsilon$ with a component perpendicular to the free surface. These waves appear when the driving amplitude exceeds a ( parameter dependent) threshold and have a frequency half that of the driving frequency. They are examples of parametric excitations in which a system exhibits motions due to a change in parameters with time. Mathematically, such systems are governed by differential equations with time varying coefficients. These are to be contrasted with the more familiar oscillatory systems, for example in mechanics, where the excitations appear as inhomogeneties in the governing differential equations. One important consequence of this difference is that a large response is evoked in the latter systems only if the driving frequency is close to one of the natural frequencies of the system whereas a parametric excitation can produce a large response also if the driving frequency is close to the harmonics and subharmonics of the natural frequencies of the system. Other familiar examples of parametric excitations are oscillations of a child's swing and those of a pendulum with oscillating point of support. A Faraday wave appears mainly due to a modulation of the gravitational field in time. Viscosity, and to a lesser extent, surface tension modify the appearance and characteristics of these waves in subtle and important ways but it is basically an inviscid phenomenon.

Faraday waves are named for Sir Michael Faraday, who first systematically observed and recorded them in 1831; his observations included the subharmonic nature of the oscillations and the appearance of square cells on the free surface. However, a discrepancy between these observations and those of Mathiessen in 1868 (cf. Douady 1990), who observed synchronous oscillations, led Rayleigh(1883) to conduct further experiments; these 
supported Faraday's observations. Theoretical investigations did not really gain momentum till the development of the theory of periodic differential equations, in particular that of Mathieu functions, first by Mathieu (1868) in the context of vibrating ellpitical membranes and Hill (1886) in the study of lunar motions and later, in the mid-twentieth century by McLachlan (1947). Benjamin \& Ursell (1954) (BU54 hereafter) were the first to show that an analysis of inviscid Faraday waves in a circular cylinder led directly to the standard Mathieu equation; the free surface was expanded in a series of horizontal spatial eigenmodes which decouple and satisfy independent Mathieu equations. For each mode, a standard Floquet analysis shows that, stable and unstable regions (the so-called 'resonance tongues') alternate in the $\Omega-\epsilon$ plane; these regions are separated by curves on which the solutions are harmonic or subharmonic, thus reconciling Faraday's and Matthiesen's observations.

BU54 also presented experimental results for the $(2,1)$ mode (two nodal circles and one nodal diameter); for a given driving frequency, good agreement in the threshold amplitude for Faraday waves to appear, between the theoretical and experimental values was reported. However, theory underpredicted the damping rate by almost a factor of 20 , as compared to experiment. BU54 hypothesised that a greater part of the dissipation was due, not to the neglected viscous effects, but to the contact line effects that were not taken into account; the contact line was assumed free to move, with the contact angle fixed at $90^{\circ}$. To avoid contact line effects, Douady (1990) performed experiments in a rectangular container with the contact line kept pinned. The effects of a meniscus were studied by performing experiments with oil (meniscus present) and water (absent); in the latter case the container was filled to the brim. The meniscus would, when in vertical oscillation as in the present case, emit surface waves at the driving frequency and could perturb the parametric standing Faraday waves. By symmetry arguments, an amplitude equation was derived for the unstable mode; its predictions were found to be in good agreement with experimental observations. Bechhoefer et al (1995) is another predominantly experimental study that explores a variety of sidewall boundaries ranging from the straight wall to sloping and shelved sides. The main idea was to keep the meniscus waves to a minimum; brimful conditions, which keep the contact line fixed, are ideal as they emit no meniscus waves but difficult to realise experimentally, according to the authors and hence not pursued much.

Viscous effects have been considered in various theoretical studies. Kumar \& Tuckerman (1994) used Floquet analysis to solve the viscous problem numerically and showed that a heuristic introduction of a linear damping term, which results in a damped Mathieu equation, does not predict the stability threshold correctly; the dissipation is over(under)estimated at higher(lower) drive frequencies. However, the equations for each horizontal spatial mode are uncoupled from the others, just as in the approach followed by BU54, even though the equations are not the Mathieu equations. One advantage of the phenomenological approach is that a set of uncoupled Mathieu equations result, just as in the inviscid case, albeit with a damping term included. Along somewhat similar lines, in an attempt to include viscous effects, Beyer \& Friedrich (1995) derived an integrodifferential equation that generalised Mathieu's equation; they however used free-slip conditions on the bottom and thus could not account for the bottom contribution to the dissipation. For weak dissipation, Muller et al (1997) present a stability analysis, using a perturbation expansion, which shows that the damped Mathieu equation has two additional memory terms representing contributions from the top and bottom viscous boundary layers. The onset amplitude, obtained from this analysis, is in much better agreement with the exact numerical solution. Moreover, a harmonic response was observed under certain conditions; this was earlier predicted numerically by Kumar (1996). 
Cerda \& Tirapegui (1998) showed that a Mathieu equation can be derived for the case of strong viscosity; this equation is different from that for the weakly viscous case and it is found that the instability mechanism is similar to the Rayleigh-Taylor instability. In all these cases, the effects of lateral walls, and in particular, of contact line conditions on those walls, was neglected.

A significant amount of experimental work on various aspects of Faraday waves has been reported in literature. Viscous effects have been shown to be important in the selection of patterns that form on the free surface; a square pattern, for example, is the observed one in low-viscosity experiments whereas the pattern changes to parallel lines in a rectangular geometry (bowed pattern in a circular geometry) for larger viscosities. Effects of finite container size have been shown to be more important for low-viscosity fluids than for high-viscosity ones (Edwards \& Fauve 1993, 1994; Bechhoefer et al 1995). Complex patterns and quasi-patterns have been reported on usage of polychromatic excitations (Edwards \& Fauve 1993). Recently, Lam \& Caps (2011) have experimentally studied the effect of a capillary meniscus on the Faraday instability threshold.

Comparison between theory and experiment have often used fit parameters. Kumar \& Tuckerman (1994) demonstrated good agreement with the experiments of Edwards \& Fauve (1994) by using the surface tension and viscosity as fitting parameters. Christiansen et al. (1995) used the slip length as a parameter to fit their experimental data; good agreement with the theory was found for slip length values that were 10 - 100 times higher than an estimate given by Milner (1991), whose formula they were using. Lam \& Caps (2011) added a phenomenological term to the linear theory for modelling the capillary effect due to the finite geometry. Along with the use of an empirical factor, this was shown to result in a quantitative agreement between theory and experiment, except for the smallest container, where the deviation was quite large. Experimental measurements of the damping rate produced values that were much larger than the values given by the ad hoc phenomenological model.

In spite of the large literature on the subject, it appears that a basic study of the effects of the contact line on the amplitude threshold of Faraday waves has not been undertaken. In this work, we take the first steps in this direction by examining the effect of a pinned contact line on the threshold for the appearance of Faraday waves; we solve the linear stability problem for a free surface in a vertically oscillating circular cylinder. We undertake this study for the inviscid case not only to understand the contact line effects in the simplest possible setting but also because it is known from experiments that wall effects, in particular the contact line conditions, are more important for the low-viscosity case. The effects of surface tension are included. For large Bond numbers, even though the stability diagrams and the subharmonic and harmonic resonances for the free and pinned contact lines are similar, the free surface shapes (for e.g. figure 7a,b) can be quite different. We follow broadly the procedure of BU54, which in the present case leads to coupled Mathieu equations governing the eigenmode dynamics. This is in contrast to the problem considered in BU54, which treated a freely moving contact line, and in Kumar \& Tuckerman (1994), Beyer \& Friedrich (1995) and Cerda \& Tirapegui (1998), who considered lateral domains of infinite extent; the dynamics of each mode was decoupled from the others and was governed by the standard uncoupled Mathieu equation or its variants. Coupled Mathieu equations have also been studied in the general literature, though not as extensively as the uncoupled system. Hansen (1985), though a little dated, has a nice discussion of the peculiarities of the coupled Mathieu system, in particular the new resonances that can occur for the so-called combination frequencies and their subharmonics. More recent work involving two coupled Mathieu equations 


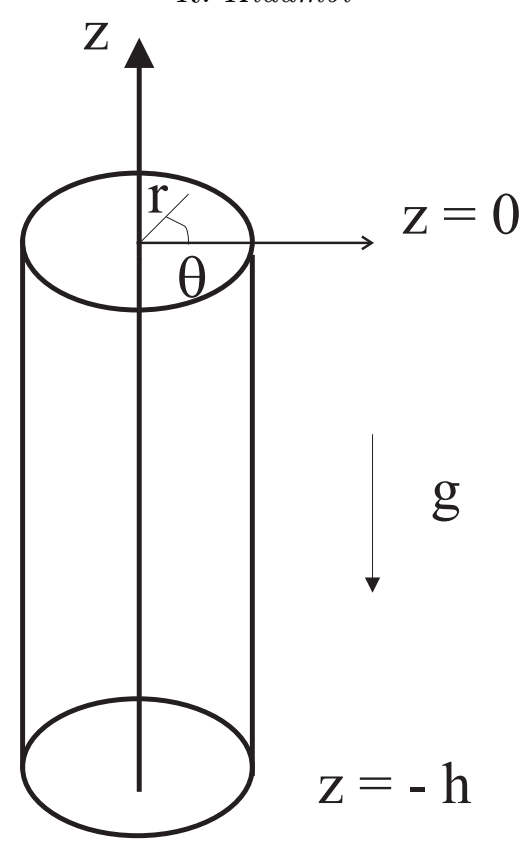

Figure 1. Geometry for Faraday waves in a circular cylinder. Liquid fills the container to a non-dimensional depth $h$, the contact line is pinned and gravity acts downward.

are Bhattacherjee (2008) which considers Faraday instability in a two-component BoseEinstein condensate and Ozakin \& Shaikh (2011), a stability analysis of surface ion traps.

This paper is organised as follows. The inviscid governing equations are presented in Sec 2. Assumption of a modal solution, followed by Galerkin projection, leads to an infinite system of coupled Mathieu equations. This, along with some popular methods to solve it, is described in Sec 3. Next, we describe the standard Hill's determinant or infinite determinant procedure to identify the harmonic and subharmonic resonances; this will be used to produce the stability diagrams in the $\Omega-\epsilon$ plane. $\S 3.2$ outlines the procedure to calculate the so-called combination resonance tongues; being non-periodic, these are invisible to the infinite determinant method. In $\S 4$, we present results for the free contact line case and the pinned contact line case for a variety of Bond numbers. Results include stability plots, shapes of the lowest modes for the harmonic and subharmonic resonances and that of the free surface at a few times. We also map the eigenvalues of the period mapping (to be described in §3.1) and relate eigenvalue collisions to harmonic, subharmonic and combination resonances.

\section{Governing equations}

We consider the motion of an inviscid liquid contained in a circular cylinder of radius $R$ subjected to vertical oscillations in the axial direction. Gravity acts along the axial direction (figure 1). The liquid depth is $H$ and its density and surface tension are respectively $\rho$ and $\sigma ; g$ is the acceleration due to gravity. The density of the fluid above the liquid is assumed negligible in comparison with that of the liquid. We scale lengths by $R$ and time by $\sqrt{R / g}$. We consider sinusoidal oscillations of the container and assume that the contact line (the intersection of the liquid surface and the lateral wall) remains pinned during the course of the motion.

We now consider the linear stability of the rest state. Assuming the deformed free 
surface to be given by $z=\eta(r, \theta, t)$ and linearising around the rest state, we have the equations governing the flow in the liquid given by

$$
\begin{gathered}
\nabla^{2} \phi=0, \phi_{r}(r=1)=0, \phi_{z}(z=-h)=0, \\
\eta_{t}=\phi_{z}, \phi_{t}+\eta(1-\epsilon \cos \Omega t)-\frac{1}{B o}\left(\eta_{r r}+\frac{\eta_{r}}{r}+\frac{\eta_{\theta \theta}}{r^{2}}\right)=0, \text { on } \mathrm{z}=0, \\
\eta(r=1, \theta, t)=0, \int_{-h}^{0} \int_{0}^{2 \pi} \eta(r, \theta, t) r d r d \theta=0 .
\end{gathered}
$$

Here $\phi$ is the velocity potential governing the flow, $h=H / R$ and $B o=\rho g R^{2} / \sigma$ is the Bond number. The normal-velocity boundary conditions on the lateral and bottom walls are given by $1(\mathrm{~b}, \mathrm{c})$ and the kinematic and normal stress conditions on the free surface by $1(\mathrm{~d}, \mathrm{e})$. Note that, under linearisation, $1(\mathrm{~d}, \mathrm{e})$ are applied on $z=0$. The pinned contact line condition is given by $1(\mathrm{f})$ and the volume conservation condition by $1(\mathrm{~g})$.

\section{Solution}

We seek $\eta$ and $\phi$ as

$\eta(r, \theta, t)=\cos m \theta \sum_{n=1}^{\infty} a_{n}(t) J_{m}\left(\delta_{m n} r\right), \phi(r, \theta, z, t)=\cos m \theta \sum_{n=1}^{\infty} b_{n}(t) \frac{\cosh k_{m n}(z+h)}{\sinh k_{m n} h} J_{m}\left(k_{m n} r\right)$

where $\delta_{m n}, k_{m n}$ are given by the roots of $J_{m}$ and $J_{m}^{\prime}$ respectively. $J_{m}$ is the Bessel function of the first kind of order $m$. Note that these forms satisfy $1(\mathrm{a}, \mathrm{b}, \mathrm{c})$ and 1 (f).

Using $2(\mathrm{a}, \mathrm{b})$ in $1(\mathrm{~d}, \mathrm{e})$, we obtain

$$
\begin{gathered}
\sum_{n=1}^{\infty}\left[\frac{d a_{n}}{d t} J_{m}\left(\delta_{m n} r\right)-k_{m n} b_{n} J_{m}\left(k_{m n} r\right)\right]=0 \\
\sum_{n=1}^{\infty}\left[\frac{d b_{n}}{d t} \operatorname{coth} k_{m n} h J_{m}\left(k_{m n} r\right)+a_{n}\left(1+\frac{\delta_{m n}^{2}}{B o}-\epsilon \cos \Omega t\right) J_{m}\left(\delta_{m n} r\right)\right]=0 .
\end{gathered}
$$

Note that, for a freely moving contact line, $\delta_{m n}=k_{m n}$, the modes decouple and $3(\mathrm{a}, \mathrm{b})$ can be combined into a single equation for $a_{n}$. Defining

$$
p_{n}=\frac{4 k_{m n} \tanh k_{m n} h}{\Omega^{2}}\left[1+\frac{\delta_{m n}^{2}}{B o}\right], q_{n}=\frac{2 \epsilon k_{m n} \tanh k_{m n} h}{\Omega^{2}},
$$

we obtain, with the change of variable $T=\Omega t / 2$, equation (2.14) of BU54, reproduced below for the reader's convenience -

$$
\frac{d^{2} a_{n}}{d T^{2}}+\left(p_{n}-2 q_{n} \cos 2 T\right) a_{n}=0 .
$$

This is the standard form of the Mathieu equation used by McLachlan (1947).

The present case, with the pinned contact line, is more complex. $3(\mathrm{a}, \mathrm{b})$ preclude a simple decoupling of the modes and it is easy to see that each $a_{n}$ is a function of all the $b_{n}$ and vice versa. Projecting $3(\mathrm{a})$ onto the test function space $\left\{J_{m}\left(\delta_{m n}\right)\right\}, n=1,2, \cdots$ via the inner product $\langle f, g\rangle=\int_{0}^{1} r f(r) g(r) d r$, we obtain

$$
\frac{d a_{n}}{d t}=\sum \alpha_{n l} b_{l}, \text { with } \alpha_{n l}=\int_{0}^{1} r k_{m l} J_{m}\left(k_{m l} r\right) J_{m}\left(\delta_{m n} r\right) d r .
$$


Inverting (4) yields

$$
b_{n}=\sum \beta_{n l} \frac{d a_{l}}{d t}
$$

which on being used in $3(\mathrm{~b})$ produces

$$
\sum_{n=1}^{N}\left[\operatorname{coth} k_{m n} h J_{m}\left(k_{m n} r\right) \sum_{l=1}^{N} \beta_{n l} \frac{d^{2} a_{l}}{d t^{2}}+a_{n}\left(1+\frac{\delta_{m n}^{2}}{B o}-\epsilon \cos \Omega t\right) J_{m}\left(\delta_{m n} r\right)\right]=0 .
$$

As is customary, we have kept only a finite number of modes for $N$ for numerical computation. Projecting (6) onto $\left\{J_{m}\left(\delta_{m p} r\right)\right\}$, we obtain the system of $N$ coupled second order ODES

$$
\sum_{n=1}^{N} \gamma_{p n} \sum_{l=1}^{N} \beta_{n l} \frac{d^{2} a_{l}}{d t^{2}}+a_{p}\left(1+\frac{\delta_{m p}^{2}}{B o}-\epsilon \cos \Omega t\right)=0, p=1,2, \cdots, N
$$

with $\gamma_{p n}=\operatorname{coth} k_{m n} h \int_{0}^{1} r J_{m}\left(k_{m n} r\right) J_{m}\left(\delta_{m p} r\right) d r$. (7) can be written in the matrix form

$$
A \frac{d^{2} y}{d t^{2}}+(\hat{P}-2 \hat{Q} \cos \Omega t) y=0
$$

where $y$ is a vector with components $a_{n}, n=1, \cdots N$; the components of $A, \hat{P}$ and $\hat{Q}$ are given by

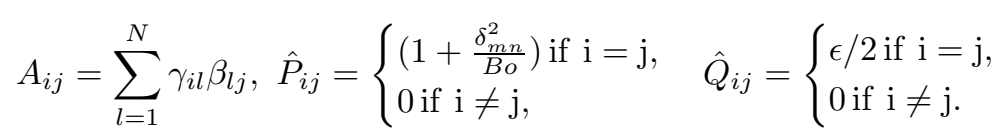

Defining $T=\Omega t / 2, P=4 A^{-1} \hat{P} / \Omega^{2}, Q=4 A^{-1} \hat{Q} / \Omega^{2}$, equation (8) can be put in a form similar to the standard Mathieu equation

$$
\frac{d^{2} y}{d T^{2}}+(P-2 Q \cos 2 T) y=0
$$

For the free contact line case considered in BU54, $P$ and $Q$ are diagonal and (9) reduces to $(2.14)$ of BU54.

(9) is a well-studied equation and there are, in general, three popular methods (Nayfeh \& Mook 1995) for its solution -

(a) Mapping at a period.

(b) Method of multiple scales.

(c) Hill's infinite determinant method.

Floquet theory gives the method of mapping at a period. To this end, (9) is written as a set of $2 \mathrm{~N}$ first order equations $\dot{y}=B(T) y$, where $B$ is a function of $P$ and $Q$ and is periodic with period $\pi$. It is well-known from Floquet theory (for e.g. Nayfeh \& Mook $1995)$ that the properties of the fundamental matrix $Z(T)$ can be used to evaluate the stability of solutions. We recall that $Z(T)$ satisfies

$$
\dot{Z}=B(T) Z, Z(0)=I,
$$

where $I$ is the identity matrix. Determination of the stability then consists of

(a) Forming $B(T)$ for the given set of parameters.

(b) Integrating (*) from $T=0$ to $T=\pi$ to obtain $Z(\pi)$.

(c) Finding the $2 N$ eigenvalues $\lambda_{i}$ of $Z(\pi)$.

(d) Establishing the stability of solutions. From Floquet theory, we have stable (resp. unstable) solutions iff $\max \left(\left|\lambda_{i}\right|\right) \leqslant 1$ (resp. $>1$ ). 
The procedure is straightforward but tedious to use in cases like the present as the calculations have to be repeated for each set of parameters. The infinite determinant method, to be described later, avoids the integrations and only the eigenvalues of certain matrices need to be found. Nevertheless, we use mapping at a period to obtain the stability boundaries issuing from the so-called combination frequencies and their sub(harmonics), for which the infinite determinant method fails.

We recall some basic facts about the eigenvalues $\lambda_{i}$. (9) is a canonical system (Lindh $\&$ Likins 1970) and Liapunov's reciprocal root theorem applies. The theorem states that if a canonical system has an eigenvalue $\lambda_{i}$, it also must have an eigenvalue $1 / \lambda_{i}$. This immediately implies instability for $\lambda_{i} \neq \pm 1$ as either $\lambda_{i}$ or its reciprocal must have modulus greater than 1 . Thus one type of stability transition boundaries are marked by the collision of two eigenvalues at \pm 1 ; it is easy to show that the plus sign corresponds to harmonic oscillations and the minus sign to subharmonic ones. However, this is not the only possible transition boundary. Instability can also be obtained in the absence of real roots. Since the roots occur as complex conjugates, a collision of two of the eigenvalues on the unit circle can cause one to move outside, leading to a modulus greater than unity and consequent instability. In this case, the eigenvalues occur with multiplicity two. This case is connected with the occurrence of combination resonances in a system and will be illustrated for the case of finite Bond numbers later.

An approximate analytic solution can be obtained as a perturbation expansion in the small parameter $\epsilon$; a naive expansion will result in secular growth and a popular way to avoid it is to use the method of multiple scales (Nayfeh \& Mook 1995, Ozakin $\&$ Shaikh 2011). The procedure involves expanding not only the solution, but also the independent variable, in a series in $\epsilon$. This results in a set of ODEs at each order and the solution is found by requiring that secular terms do not appear. The method is useful for producing analytic approximations to the stability boundaries for small $\epsilon$ but is not otherwise suitable for explorations of a large part of the $\Omega-\epsilon$ space. Not only are the calculations involved highly cumbersome but also it is difficult (Hansen 1985) to guess the right number of terms to include, with computations at different orders producing different stability boundaries (figure 1 of Hansen 1985). We mention the method only for completeness and will not be using it in our computations.

In this paper, we will be mainly using the infinite determinant method, which is briefly described in the next section.

\subsection{The infinite determinant method}

The infinite determinant method, first introduced by Hill (1886) in the study of perturbed planetary motions, is based on the Floquet theorem which we recall -

Consider the system of ODEs, introduced in the last section, $\dot{y}=B(T) y$ where $B(T)$ is a periodic matrix of period $\pi$. Let $\rho$ be a characteristic (Floquet) multiplier and let $\mu$ be the corresponding characteristic exponent so that $\rho=e^{\mu \pi}$. Solutions $y(t)$ of $\dot{y}=B(T) y$ are of the form $y(T)=e^{\mu T} p(T)$, where $p(T+\pi)=p(T)$. This implies that $y(t+\pi)=\rho y(t)$. In particular, $p(t)$ can be expanded in a Fourier series and the solution to (9) can be sought as

$$
y(T)=e^{\mu T}\left\{\sum_{l=0}^{L} \xi_{l} e^{i l 2 T}+\sum_{l=1}^{L} \chi_{l} e^{-i l 2 T}\right\} .
$$

$\xi_{l}$ and $\chi_{l}$ are vectors with components $\xi_{l}^{n}, \chi_{l}^{n}, n=1, \cdots N$. It is easy to see from equation (10) that we have a stable (unstable) solution if $|\rho|<1(>1$ ) for some parameter values. Assuming continuous dependence of $\rho$ on the parameters, it follows that $|\rho|=1$ on 
curves demarcating the stable and unstable regions. Now $\rho$ can be real or complex on these curves; in particular, for $\rho= \pm 1$, we have periodic solutions of period $\pi$ and $2 \pi$ respectively. These are the harmonic and subharmonic oscillations and correspond respectively to $\mu=0$ and $\mu=i$. Complex values of $\rho$ on the unit circle lead to almost periodic solutions; Kumar \& Tuckerman (1994) claim that, for the Faraday problem that they consider, these never produce growing solutions and hence need not be considered in the analysis.

For unforced oscillations $(\epsilon=Q=0), y(T)=\cos n T, n=1,2, \cdots$ is the solution corresponding to a quiescent initial state. It is easily checked that odd (even) $n$ produce the subharmonic (harmonic) oscillations, with attendant natural frequencies which can be continued to higher $\epsilon$. This process produces curves in the $\Omega-\epsilon$ plane, on which the oscillations are either harmonic or subharmonic and which are also the boundaries separating the regions of stable oscillations from those of unstable ones. The regions separated by these boundaries are the so-called fundamental resonance tongues (FRTs) or Mathieu tongues; the bottoms of these tongues correspond to $\epsilon=0$. For the free contact line considered in BU54, the frequencies are simply given by $\omega_{n}=2 \omega_{i j} / n$ with $\omega_{i j}^{2}=k_{i j} \tanh k_{i j} h\left(1+\delta_{i j}^{2} / B o\right)$ is the natural frequency of the $(\mathrm{i}, \mathrm{j})$ mode. For the pinned contact line, the object of the present study, the unforced frequencies cannot be expressed as a closed form expression and must be numerically obtained by solving an eigenvalue problem (for e.g. Kidambi \& Shankar 2004).

Plugging (10) in (9), we have the $m^{\text {th }}$ equation of the system (9) given by

$$
\begin{gathered}
\sum_{l=1}^{L-1}\left[\xi_{l}^{m}\left(\mu^{2}+4 i \mu l-4 l^{2}\right)+\sum_{n=1}^{N}\left\{P_{m n} \xi_{l}^{n}-Q_{m n}\left(\xi_{l-1}^{n}+\xi_{l+1}^{n}\right)\right\}\right] e^{i l 2 T} \\
+\sum_{l=2}^{L-1}\left[\chi_{l}^{m}\left(\mu^{2}-4 i \mu l-4 l^{2}\right)+\sum_{n=1}^{N}\left\{P_{m n} \chi_{l}^{n}-Q_{m n}\left(\chi_{l-1}^{n}+\chi_{l+1}^{n}\right)\right\}\right] e^{-i l 2 T} \\
+\left[\chi_{1}^{m}\left(\mu^{2}-4 i \mu-4\right)+\sum_{n=1}^{N}\left\{P_{m n} \chi_{1}^{n}-Q_{m n}\left(\xi_{0}^{n}+\chi_{2}^{n}\right)\right\}\right] e^{-i 2 T}+\xi_{0}^{m} \mu^{2}+\sum_{n=1}^{N}\left[P_{m n} \xi_{0}^{n}-Q_{m n}\left(\xi_{1}^{n}+\chi_{1}^{n}\right)\right] \\
+\left[\xi_{L}^{m}\left(\mu^{2}+4 i \mu L-4 L^{2}\right)+\sum_{n=1}^{N}\left\{P_{m n} \xi_{L}^{n}-Q_{m n} \xi_{L-1}^{n}\right\}\right] e^{i L 2 T} \\
{\left[\chi_{L}^{m}\left(\mu^{2}-4 i \mu L-4 L^{2}\right)+\sum_{n=1}^{N}\left\{P_{m n} \chi_{L}^{n}-Q_{m n} \chi_{L-1}^{n}\right\}\right] e^{-i L 2 T}=0}
\end{gathered}
$$

Since the Fourier modes are independent, the coefficients of $e^{i l 2 T}$ have to vanish for $l=-L, \cdots, L$. This leads to the following system of $(2 L+1)$ equations -

$$
\begin{gathered}
\xi_{0}^{m} \mu^{2}+\sum_{n=1}^{N}\left[P_{m n} \xi_{0}^{n}-Q_{m n}\left(\xi_{1}^{n}+\chi_{1}^{n}\right)\right]=0 \\
{\left[\xi_{l}^{m}\left(\mu^{2}+4 i \mu l-4 l^{2}\right)+\sum_{n=1}^{N}\left\{P_{m n} \xi_{l}^{n}-Q_{m n}\left(\xi_{l-1}^{n}+\xi_{l+1}^{n}\right)\right\}\right]=0, l=1, \cdots L-1} \\
{\left[\chi_{1}^{m}\left(\mu^{2}-4 i \mu-4\right)+\sum_{n=1}^{N}\left\{P_{m n} \chi_{1}^{n}-Q_{m n}\left(\xi_{0}^{n}+\chi_{2}^{n}\right)\right\}\right]=0}
\end{gathered}
$$




$$
\begin{gathered}
{\left[\chi_{l}^{m}\left(\mu^{2}-4 i \mu l-4 l^{2}\right)+\sum_{n=1}^{N}\left\{P_{m n} \chi_{l}^{n}-Q_{m n}\left(\chi_{l-1}^{n}+\chi_{l+1}^{n}\right)\right\}\right]=0, l=2, \cdots L-1} \\
{\left[\xi_{L}^{m}\left(\mu^{2}+4 i \mu L-4 L^{2}\right)+\sum_{n=1}^{N}\left\{P_{m n} \xi_{L}^{n}-Q_{m n} \xi_{L-1}^{n}\right\}\right]=0} \\
\\
{\left[\chi_{L}^{m}\left(\mu^{2}-4 i \mu L-4 L^{2}\right)+\sum_{n=1}^{N}\left\{P_{m n} \chi_{L}^{n}-Q_{m n} \chi_{L-1}^{n}\right\}\right]=0}
\end{gathered}
$$

This system is just from the $m^{\text {th }}$ equation of (9). Hence, (9) leads to a system of $(2 L+1) \times N$ equations. Traditionally, in linear stability analyses, an eigenvalue problem is solved for the temporal growth rate, in this case $\mu$. However, $\mu$ occurs quadratically in the eigenvalue system; it is simpler to pose an eigenvalue problem for the driving amplitude $\epsilon$ which occurs linearly in $Q$. Thus, for given $\Omega$, we fix $\mu=0$ (the harmonic case) or $\mu=i$ (the subharmonic case) and solve for $\epsilon$. This is also the approach of Kumar \& Tuckerman (1994) for the laterally unbounded viscous problem. Moving the $Q$ terms to the right hand side in (12), we obtain a generalised eigenvalue problem

$$
C v=\epsilon D v
$$

where $v$ is a vector with $(2 L+1) N$ components $\xi_{0}^{1}, \cdots \xi_{L}^{1}, \chi_{1}^{1}, \cdots \chi_{L}^{1}, \cdots \xi_{0}^{N}, \cdots \xi_{L}^{N}, \chi_{1}^{N}, \cdots \chi_{L}^{N}$.

The goal of the present work is to compute the stability diagram in the $\Omega-\epsilon$ plane, for the case of the pinned contact line. To this end, for a given $\Omega$, we generate the matrices $P$ and $Q$, assume either $\mu=0$ (the harmonic case) or $\mu=i$ (the subharmonic case) and solve the resulting eigenvalue problem (13), for $\epsilon$. The LAPACK routine ZGGEV has been used to get the eigenvalues.

\subsection{Combination resonances and their calculation}

When $P$ and $Q$ are not diagonal as is the case for the pinned contact line, it is well-known (for example Nayfeh \& Mook 1995) that combination resonances can occur apart from the usual (sub)harmonic ones. These resonances occur at frequencies $\left| \pm \omega_{i} \pm \omega_{j}\right| / s, s=1,2, \cdots$ where $\omega_{i, j}$ are any two natural frequencies of the system. The tongues emanating from these, the so-called combination resonance tongues (CRTs) further shrink the stable regions. The boundaries of the CRTs cannot be found by setting $\mu=0$ or $i$, unlike for the FRTs. This is because, even though $\mu_{r}=0$ on these boundaries, the value of $\mu_{i}$ is unknown and has to be determined as part of the solution. In fact, the oscillatory states corresponding to points on the CRT boundaries are not periodic, unlike those for points on the FRT boundaries which are either harmonic or subharmonic. An example of such quasi-periodic motion for $B o=100$ is shown later in figure 17. The CRT boundaries can be determined, by the mapping at a period, and are in general found to intersect the FRTs. Since the latter enclose unstable regions, the CRTs are not continued beyond the FRT boundaries and are terminated there. Hansen (1985) gave a heuristic approach to calculate the CRTs and no rigorous method seems to be available to calculate these. We will use the mapping at a period, already described in $\S 3.1$, to find the CRTs.

\section{Results \& Discussion}

First we fix the notation for the modes. By mode $(m, n)$, we mean that the mode possesses $2 m$ azimuthal and $n$ radial nodes, not counting those on the wall. For the free contact line case, the $(m, n)$ mode is just proportional to the $n^{\text {th }}$ Bessel mode i.e. 


\begin{tabular}{|c|c|c|c|c|c|c|}
\hline \multirow{2}{*}{ Case } & \multicolumn{5}{|c|}{$\omega_{10}$} & \multicolumn{3}{|c|}{$\omega_{11}$} \\
\cline { 2 - 7 } & $B o=\infty$ & 1000 & 100 & $B o=\infty$ & 1000 & 100 \\
\hline Free $\mid$ & 1.323 & 1.325 & 1.345 & 2.309 & 2.342 & 2.617 \\
\hline Pinned $\mid 1.323$ & $\begin{array}{c}1.380(30) \\
1.339(2)\end{array}$ & $\begin{array}{c}1.537(30) \\
1.459(2)\end{array}$ & 2.309 & $\begin{array}{c}2.408(30) \\
2.354(2)\end{array}$ & $\begin{array}{c}2.883(30) \\
2.738(2)\end{array}$ \\
\hline
\end{tabular}

TABLE 1. The frequencies $\omega_{10}$ and $\omega_{11}$ for the free and pinned cases for a few Bond numbers. $h=1$.

\begin{tabular}{l}
$\overline{\left|2 \omega_{10}\right| 2 \omega_{10} / 3\left|2 \omega_{10} / 5\right| 2 \omega_{10} / 2\left|2 \omega_{10} / 4\right| 2 \omega_{10} / 6 \mid}$ \\
$\overline{|2.646| 0.882|0.529| 1.323|0.662|} 0.441 \mid$ \\
$\overline{\left|2 \omega_{11}\right| 2 \omega_{11} / 3\left|2 \omega_{11} / 5\right| 2 \omega_{11} / 2\left|2 \omega_{11} / 4\right| 2 \omega_{11} / 6 \mid}$ \\
\hline $4.618|1.539| 0.924|2.309| 1.155|0.769|$ \\
\hline
\end{tabular}

TABLE 2. The first three (sub)harmonics of $\omega_{10}$ and $\omega_{11}$ for $B o=\infty, h=1$.

$\cos m \theta J_{m}\left(\delta_{m n} r\right)$ whereas a sum of such Bessel modes is required to represent the $(m, n)$ mode in the pinned case.

To keep matters brief, we will show results mainly for the height $h=1$ and the first azimuthal mode $m=1$. We start off by tabulating the frequencies of the $(1,0)$ and $(1,1)$ modes for the free and pinned cases, for a variety of Bond numbers, in Table 1. For the pinned contact line, two modes are sufficient to obtain converged results for the infinite $B o$ case whereas a large number of modes are required to obtain converged results for the lower $B o=1000$ and 100. The frequencies computed using 30 and 2 modes are shown for the lower $B o=100,1000$; there is a maximum error of $5 \%$ between the two sets of values. Since the sub(super)hamonic and harmonic frequencies are relevant to Faraday oscillations, we tabulate these as well in Table 2 , for $B o=\infty$, the case we will predominantly consider. As the numbers are virtually identical for the free and pinned cases, only one set is shown.

We first reproduce the results for the case of the free contact line. To start with, we consider the case of no surface tension $(B o=\infty)$. We plot the driving amplitude versus the driving frequency in figures 2 and 3. Figures $2(\mathrm{a}),(\mathrm{b})$ are the stability curves for the $(1,0)$ and $(1,1)$ modes. These figures show the harmonic and subharmonic resonances excited for the respective modes and the FRTs emanating from these resonances, at $\epsilon=0$. For a given mode, the FRTs divide the plane into regions where the flow is stable or unstable; these regions alternate and are marked in the figures. The tongues corresponding to different modes overlap; this however does not affect the stability properties of the system as it is possible to excite each of these modes separately (for e.g. the $(2,1)$ mode alone is excited in BU54). In the figures, the solid and dashed lines correspond to 


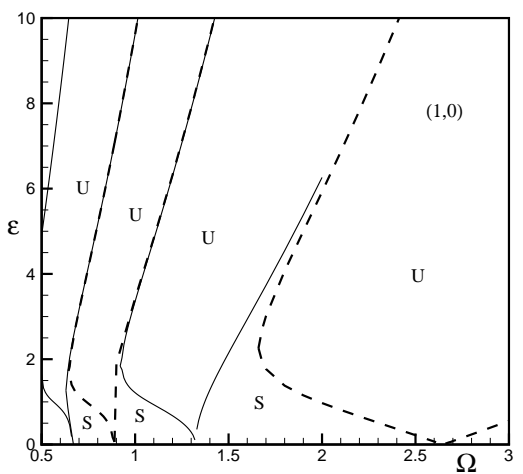

(a)

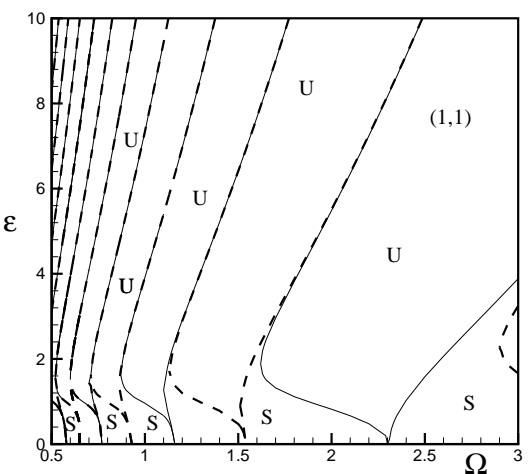

(b)

FiguRE 2. Stability plot for the a) $(1,0)$ and b) $(1,1)$ Faraday modes in a circular cylinder with free contact line. The FRTs for each of the modes are non-overlapping but overlap with those of the other. The tongue boundaries emanating from harmonic and subharmonic resonances are shown as solid and dashed lines respectively. ' $U$ ' and ' $S$ ' refer to unstable and stable regions. In (b), the fundamental subharmonic $2 \omega_{11}$ is not shown.

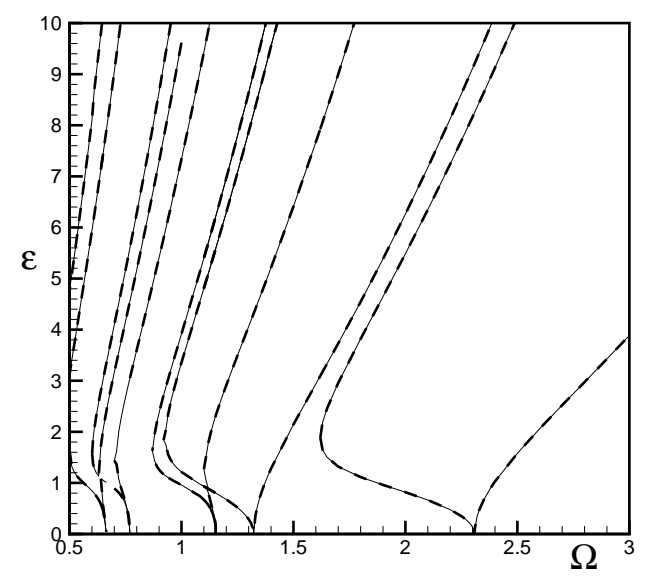

FiguRE 3 . Stability plot for the $(1,0)$ and $(1,1)$ modes in a circular cylinder with pinned contact line. Solid and dashed lines correspond to $N=3$ and $N=2$. Only the tongues corresponding to the harmonics of these modes have been shown.

harmonic and subharmonic oscillations. In figure 2(a), for example, we have the first and second subharmonic tongues emerging from $\Omega \approx 2.64$ and 0.89 respectively; the corresponding harmonic tongues emerge from $\Omega \approx 1.32$ and 0.66 respectively. These are in accordance with the values shown in Table 2. It is clear that the FRTs do not intersect and the $\Omega-\epsilon$ plane is a sum of such non-overlapping stable and unstable regions.

We next consider the case when the contact line is pinned. The analogous plot is shown in figure 3. The computations were performed with $\mathrm{N}=2,3$ and 4 and $\mathrm{L}=40$ modes 
(i.e. a total of 162, 243 and 324 modes) and convergence was checked with the solid and dashed lines in the figure corresponding to $N=3$ and 2 respectively. $N=4$ gave substantially the same curves and has not been shown. It appears that $N=2$ or 3 and $L=40$ is enough to produce converged results. However, $N=2$ will produce only the lowest two fundamental frequencies and their (sub)harmonics; computations with larger $N$ are required to obtain the higher fundamental frequencies and their (sub)harmonics. Since the values of $\omega_{10}$ and $\omega_{11}$ are virtually identical for the free and pinned cases, their stability diagrams are too. The difference lies in the fact that any pinned mode will involve all the Bessel modes and so the regions of instability will be more, unlike in the free case where each Bessel mode corresponds to a free mode and can be excited by itself. Accordingly, the free surface shapes and the velocity fields will be different. Let us consider the free surface shape $\eta$ as given by 2(a). Only $a_{1}(t)$ (resp. $a_{2}(t)$ ) contribute to the $(1,0)$ (resp. $(1,1))$ mode shapes for the free contact line whereas $a_{n}(t), n=1,2, \cdots$ contribute to all the mode shapes when the contact line is pinned. We illustrate, in figure 4 , the mode shapes for a driving frequency $\Omega=2$. From figure $2(\mathrm{a}, \mathrm{b})$, it is clear that, for $\Omega=2$ and low driving amplitudes $\epsilon$, there are two subharmonic (period $2 \pi$ ) resonances at $\epsilon \approx 0.818$ and 5.486 and one harmonic (period $\pi$ ) resonance at $\epsilon \approx 6.262$ for the $(1,0)$ mode and two harmonic (at $\epsilon \approx 0.972,5.571)$ and one subharmonic resonance (at $\epsilon \approx 5.924$ ) for the $(1,1)$ mode. Resonances at similar values will occur for the $B o=\infty$ pinned case as well. The variation of $a_{1}$ with time for the $(1,0)$ and $(1,1)$ free modes is shown in figure 4; the corresponding variations of $a_{1}$ and $a_{2}$ for the $(1,0)$ and $(1,1)$ pinned modes are shown in figures 5 and 6 . Only $a_{1}$ and $a_{2}$ are present for these cases since we are showing the results of a $N=2$ computation; a larger number of modes has to be used to establish convergence. As should be clear, the presence of the $a_{n}$ makes the $\eta$ for the pinned case very different from that for the free case even though the frequencies are virtually identical for the infinite Bond number case. This is demonstrated in figure 7 where we plot $\eta(r)$ for one harmonic and one subharmonic resonance each for the free and pinned cases. The $\eta$ for the harmonic case is plotted at $T \approx 2.07$ and that for the subharmonic case at $T=\pi / 2$.

We show the tongues issuing from the $(1,2)$ modal frequency and its first two harmonics $\omega_{12} / 2, \omega_{12} / 3$ in figure 8 . Finally we show the harmonic and subharmonic tongues for the $(1,0)$ and $(1,1)$ modes in figure 9 . In this figure, we also mark those regions of the $\Omega-\epsilon$ plane where the mapping at a period has at least one eigenvalue of magnitude greater than 1. These represent the unstable regions and they match well with the unstable regions obtained from the earlier calculations, as can be seen. The mapping at a period however is incapable of distinguishing between the various resonance tongues. The stability plots for finite $B o$ will be quite different for the free and pinned cases as the fundamental frequencies are quite different (Table 1) but otherwise the scenario is similar to those presented above. However, a larger number of modes have to be used to obtain converged results for the lower Bond numbers.

It is well-known that (Lindh \& Likins 1970) having all eigenvalues of the period mapping on the unit circle is a necessary but not sufficient condition for existence of periodic (either harmonic / subharmonic) oscillations. Depending on the argument of the eigenvalues, one could have almost periodic oscillations. A collision of eigenvalues at \pm 1 indicates a harmonic(subharmonic) oscillation i.e. there is only a pair of repeated eigenvalues. For coupled Mathieu systems, however there could be eigenvalue collisions on the unit circle off the x-axis i.e. there are at least two pairs of repeated eigenvalues. This has to do with the presence of combination resonances in the coupled system and their subharmonics i.e. for frequencies $\left| \pm \omega_{i} \pm \omega_{j}\right| / s, s=1,2, \cdots$ where $\omega_{i, j}$ are the fundamental frequencies of the coupled system. 


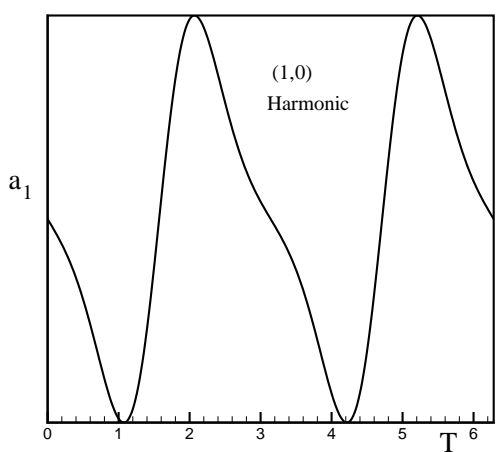

(a)

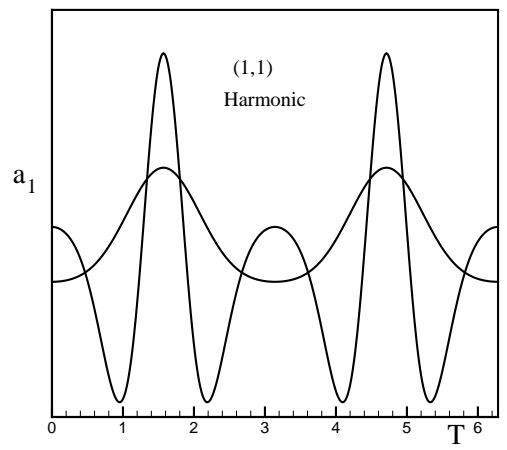

(c)

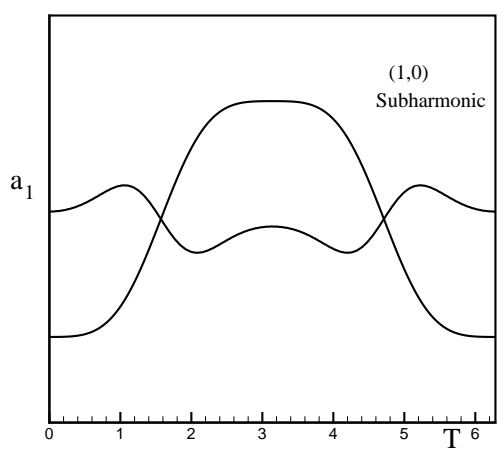

(b)

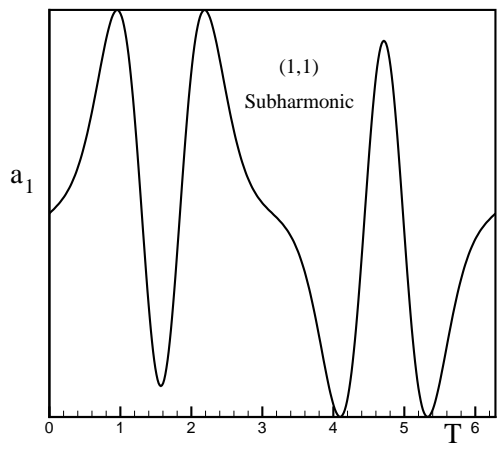

(d)

FiguRE 4 . Variation of $a_{1}$ with time for a driving frequency $\Omega=2$. The contact line is free to move. In cases (a) and (b), the (1,0) mode alone and in cases (c) and (d), the (1,1) mode alone are excited. At this driving frequency and low driving amplitudes, there is one harmonic (a) and two subharmonic (b) oscillatory configurations possible for the $(1,0)$ mode and two harmonic (c) and one subharmonic (d) configurations for the $(1,1)$ mode. $B o=\infty$.

Indeed, a key difference between the free and pinned contact line cases is the occurrence of combination resonances for the latter. This has two effects - a) the unstable regions increase and (b) the oscillatory states corresponding to these are not periodic. We show the stability plots deduced from the eigenvalues of mappings at a period for three different Bond numbers, in figure 10. In figure 10(a), for the case of infinite $B o$, the FRTs (from right to left) corresponding to $2 \omega_{10}, \omega_{11}, 2 \omega_{11} / 3, \omega_{10}$ and $2 \omega_{11} / 4$ can be discerned. The first and third frequencies are subharmonics; the remaining harmonics. There are no discernible CRTs in this case. Figure 10(b) shows the case for $B o=1000$. The FRTs are very similar to the infinite $B o$ case since the frequencies are quite similar (Table 1 ). However, one can now discern two CRTs (marked with arrows in the figure) in this case. The one on the right corresponds to the combination resonance $\left(\omega_{10}+\omega_{11}\right) / 2 \approx 1.847$ 


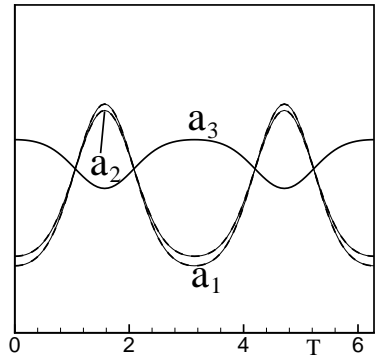

(a)

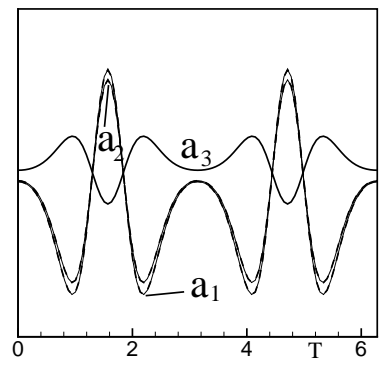

(b)

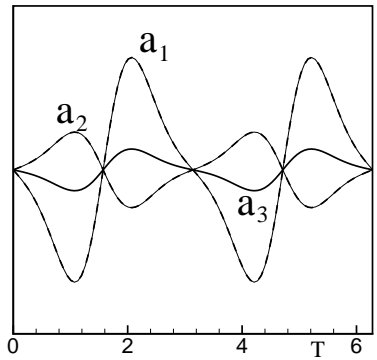

(c)

FIGURE 5. Variation of $a_{1}, a_{2}$ and $a_{3}$ with time for a driving frequency $\Omega=2$ and driving amplitude $\epsilon=$ a) 0.818 b) 5.486 and c) 6.262 . The contact line is pinned. The dashed lines are 3 mode calculations and are in good agreement with the 2 mode calculations for $a_{1}$ and $a_{2}$. At this driving frequency and low driving amplitudes, there are three harmonic oscillatory configurations possible. $B o=\infty$.

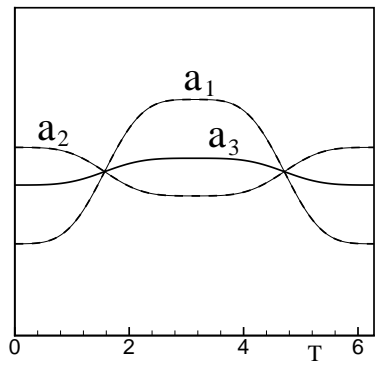

(a)

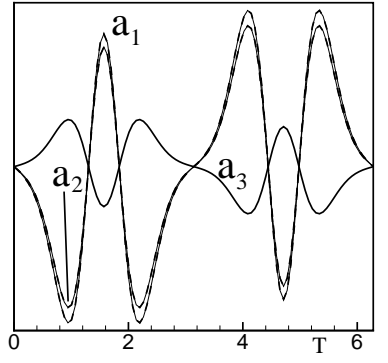

(b)

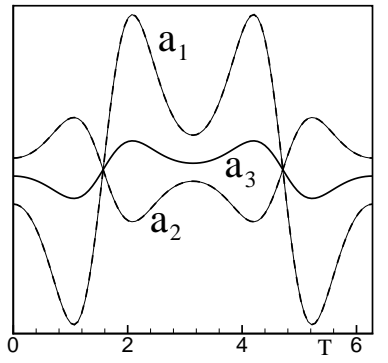

(c)

FiguRE 6. Variation of $a_{1}, a_{2}$ and $a_{3}$ with time for a driving frequency $\Omega=2$ and driving amplitude $\epsilon=$ a) $0.972 \mathrm{~b}) 5.571$ and c) 5.924 . The contact line is pinned. The dashed lines are 3 mode calculations and are in good agreement with the 2 mode calculations for $a_{1}$ and $a_{2}$. At this driving frequency and low driving amplitudes, there are three subharmonic oscillatory configurations possible. $B o=\infty$.

while the one on the left corresponds to $\left(\omega_{10}+\omega_{11}\right) / 3 \approx 1.231$. Figure $10(\mathrm{c})$ shows the situation for $B o=100$. The FRTs now emanate from appreciably different values of the driving frequency, compared with the infinite $B o$ case. Two well developed CRTs, emanating from $\omega_{10}+\omega_{11} \approx 4.197$ and $\left(\omega_{10}+\omega_{11}\right) / 2 \approx 2.099$ can be clearly distinguished.

We show some eigenvalue plots of the period mapping for the $B o=100$ and 1000 cases, in figure 11. Figure $11(\mathrm{a})$ is for $B o=1000$ and a fixed drive frequency $\Omega=1.7$ and drive amplitudes $\epsilon$ ranging from 0 to 3 ; figure $10(\mathrm{~b})$ is for $B o=100$ and $\Omega=2$. We recall that a coupled system of $N$ second order ODEs will have $2 N$ eigenvalues, occuring as complex conjugates because the coefficients of the ODE system are real. Also, their product would be unity. For the present computation, $N=2$ and we have four eigenvalues. As $\epsilon$ is varied, these fill mainly three regions - the unit circle, parts of 


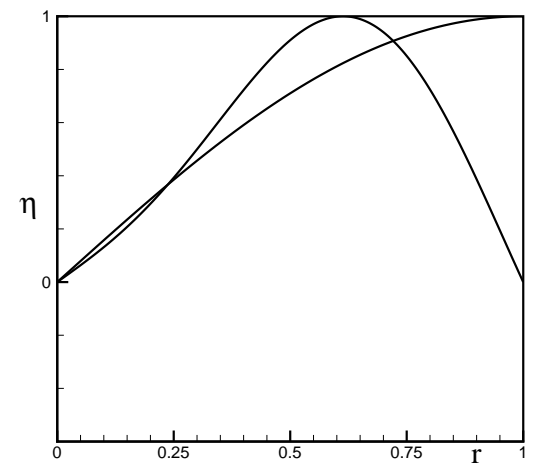

(a)

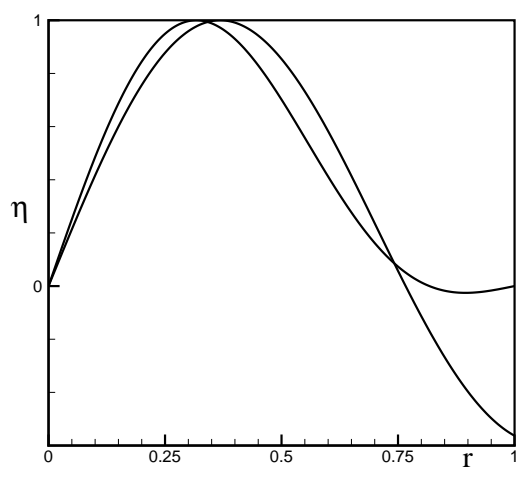

(b)

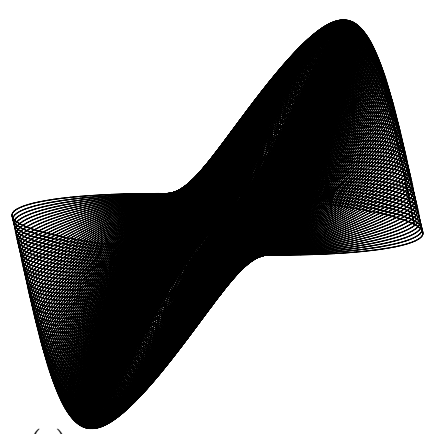

(c)

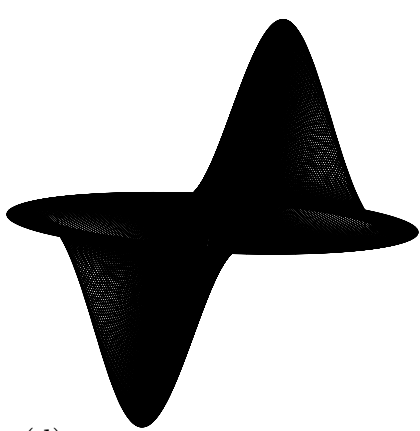

(d)

Figure 7. A plot of the free surface $\eta(r)$ for $\theta=0$; the two curves represent the free and pinned contact line cases. The driving frequency $\Omega=2$. a) The profile at $T=2.07$ for the $(1,0)$ harmonic resonance with $\epsilon=6.262$, b) The profile at $T=\pi / 2$ for the $(1,1)$ subharmonic resonance with $\epsilon=5$.571. $\eta(r=1)=0$ and $d \eta / d r(r=1)=0$ identify the pinned and free contact line cases in these figures. (c) and (d) show the free surface for the $(1,0)$ harmonic and the $(1,1)$ subharmonic pinned modes.

the real axis and two lines off the unit circle. We have stability when the eigenvalues lie on the unit circle and instability for the other cases as at least one of the eigenvalues will have modulus greater than unity. For the larger $B o$, the eigenvalues form tiny blips off the unit circle (marked by arrows in figure 11(a)) whereas these are more substantial for $B o=100$. This is in accordance with the thicker CRTs for this case as compared to $B o=1000$ (figures 10b,c).

We next show the eigenvalue plots for a few values of the driving amplitude $\epsilon$. Figure 12 is for $B o=1000$. At small $\epsilon$, the system is stable as indicated by the eigenvalues remaining on the unit circle (figure 12a). At $\epsilon \approx 0.96$, there is a collision of eigenvalues 


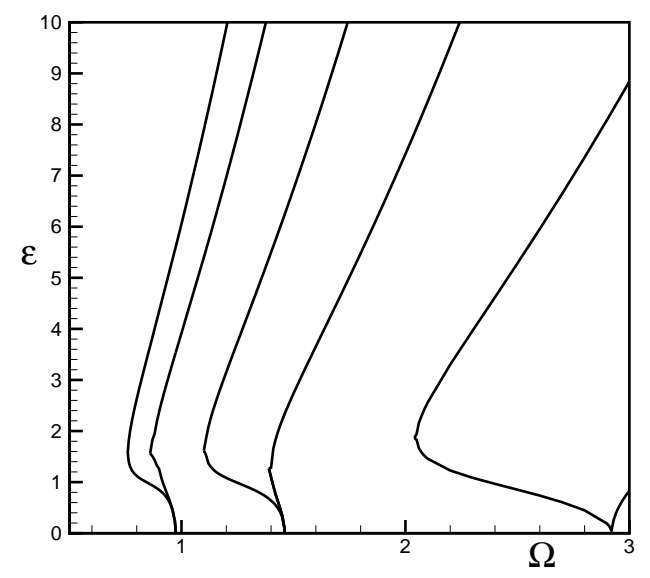

FiguRE 8 . Stability plot for the $(1,2)$ Faraday mode in a circular cylinder with pinned contact line. The tongues emanating from the $(1,2)$ modal frequency $\omega_{12}$ and its first two harmonics $\left(2 \omega_{12} / 4,2 \omega_{12} / 6\right)$ are shown.

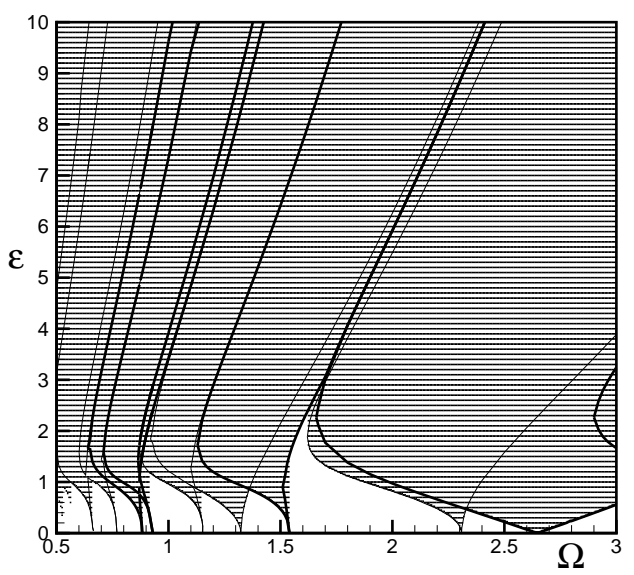

FIGURE 9. Stability plot for the first two Faraday modes in a circular cylinder with pinned contact line. Thick and thin lines represent subharmonic and harmonic oscillations respectively. $N=2$. The dotted region represents areas of the parameter space where the mapping at a period has at least one eigenvalue of modulus greater than $1 . B o=\infty$.

on the unit circle (figure 12b), off the x-axis, leading to a region of instability (the right CRT shown in fig 10(b)). The conditions on the CRT boundary correspond to almost periodic oscillations. The eigenvalues which moved off the unit circle return to it at $\epsilon \approx 1$, and this marks the end of the CRT boundary for this $\Omega$. Beyond this, there is a stable region wherein the eigenvalues again continue on the unit circle (not shown in the panels). Figure 12(c) shows the situation for $\epsilon=1.485$, by which value, two of the eigenvalues have collided at +1 and moved on to the real axis. The point of collision signifies a harmonic resonance beyond which lies the FRT, in this case, emanating from $\omega_{11}$. For still higher values of $\epsilon$, like for example 2.19 (figure 12d), the remaining pair of eigenvalues 

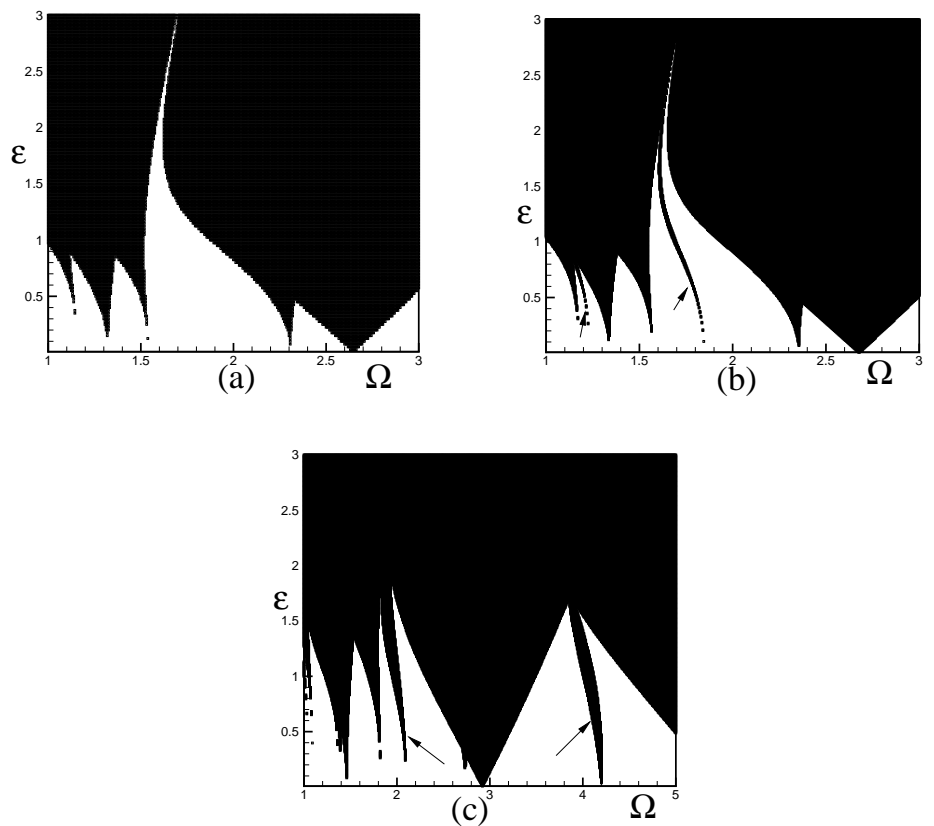

FiguRE 10. Stability plot for the first two Faraday modes in a circular cylinder with pinned contact line. (a) $B o=\infty$ (b) $B o=1000$ (c) $B o=100$. CRTs can be clearly discerned for the finite $B o$ cases.

has moved off the unit circle as well, having collided at $x=-1$; this corresponds to a subharmonic resonance which marks the boundary of the FRT emanating from $2 \omega_{10}$. Similar observations may be made for figure 13 which shows the case for $B o=100$.

Note that more modes are required for producing converged frequencies as $B o$ decreases, as can be seen from Table 1. Thus, the previous results should be taken only as qualitative, though illustrative of the general scenario. It is not difficult to compute using a larger number of modes; however, the harmonics, subharmonics and the combination resonances become so numerous that the stability diagram becomes hopelessly cluttered. As an example, we present the stability diagram for $B o=100$ obtained by using three modes, instead of two, in figure 14. Resonance tongues emanating from twelve important frequencies have been marked in the figure $-F_{1}, F_{2}$ and $F_{3}$ are the fundamental frequencies $\omega_{10}, \omega_{11}$ and $\omega_{12}, H_{1}$ is the harmonic $\omega_{12} / 2, S_{1}, S_{2}$ and $S_{3}$ are the subharmonics $2 \omega_{11} / 3,2 \omega_{12} / 3$ and $2 \omega_{10}$ and $C_{1}, C_{2}, C_{3}, C_{4}$ and $C_{5}$ are the combination resonances $\left(\omega_{10}+\omega_{11}\right) / 2,\left(\omega_{11}+\omega_{12}\right) / 3,\left(\omega_{10}+\omega_{12}\right) / 2,\left(\omega_{11}+\omega_{12}\right) / 2$ and $\omega_{10}+\omega_{11}$. With a three mode calculation, $\omega_{10}, \omega_{11}$ and $\omega_{12}$ turn out to be respectively $1.486,2.792$ and 4.015 .

Figure 15 shows an eigenvalue plot of the period mapping computed using three modes. The excursions off the unit circle point to the occurrence of combination resonances which clearly are much more numerous than when only two modes were used. To get a better understanding, we examine the plots for a fixed $\Omega$. In figure 16 , we show the eigenvalue plot for a fixed value of $\Omega=2.12 ; 16$ (a) shows all the eigenvalues for $\epsilon$ ranging from 0 to 3 whereas (b), (c) and (d) show the eigenvalues for particular values of $\epsilon=0.3225,1.3125$ and 1.44 respectively. Since we have used 3 modes, we have six eigenvalues for any given drive amplitude $\epsilon$. From figure 16(a), it is clear that there are two CRTs occuring at this 


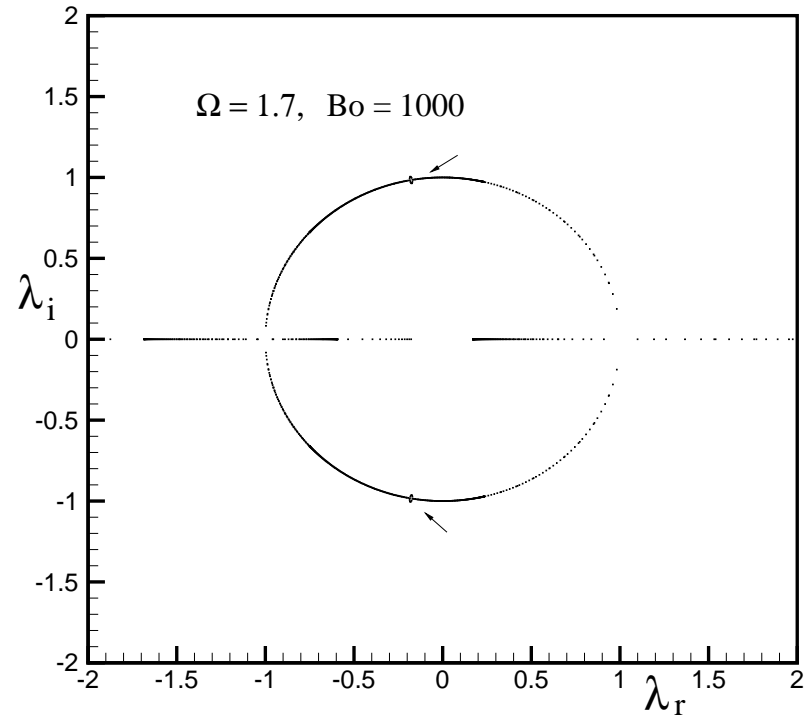

(a)

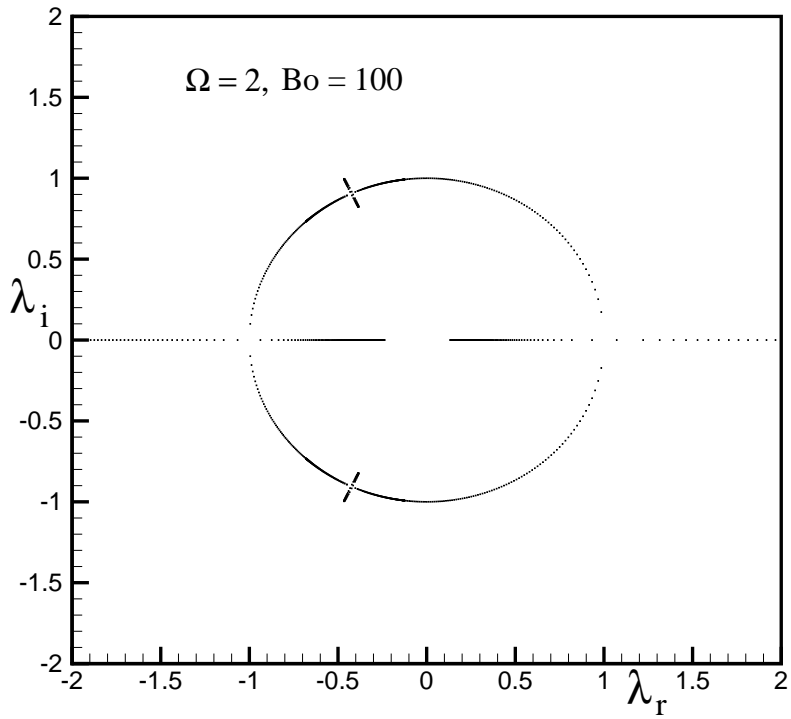

(b)

FiguRE 11. Eigenvalues of the mapping at a period. (a) $B o=1000, \Omega=1.7$ (b) $B o=100, \Omega=2$. The driving amplitude $\epsilon$ ranges from 0 to 3 . 


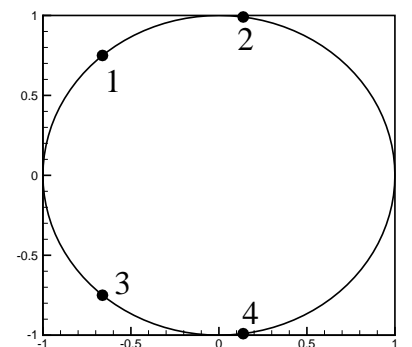

(a)

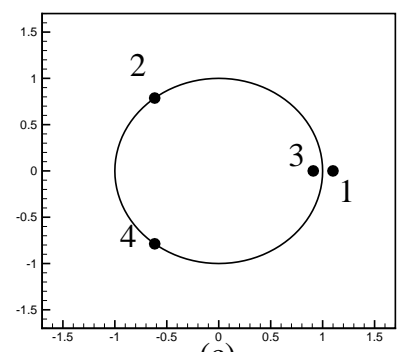

(c)

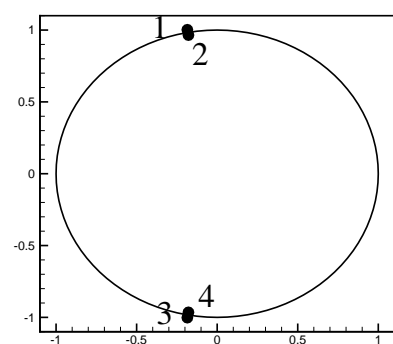

(b)

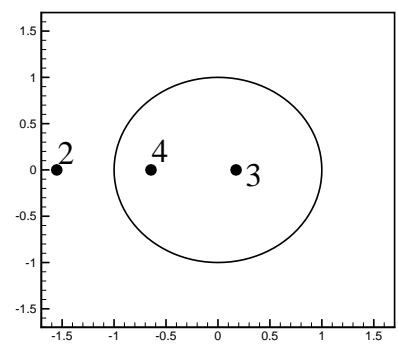

(d)

Figure 12. Eigenvalues of the mapping at a period for $\Omega=1.7, B o=1000 . \epsilon=\mathrm{a}) 0.45 \mathrm{~b}) 0.96$ c) 1.485 and d) 2.19 . In (d), eigenvalue ' 1 ' has gone beyond the range of the figure, to the right, and hence is not seen.

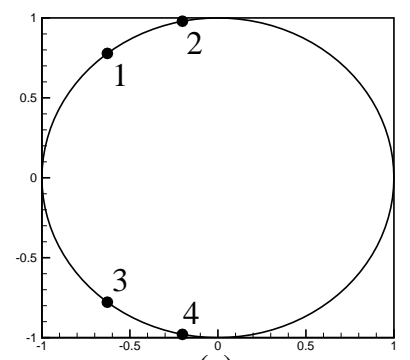

(a)

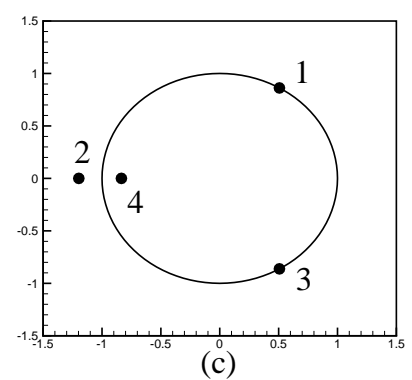

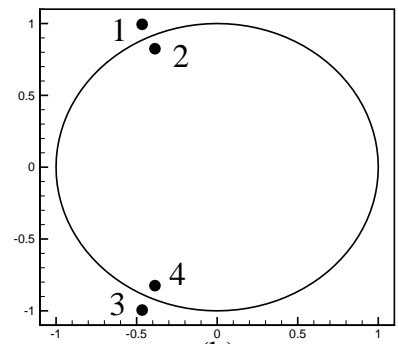

(b)

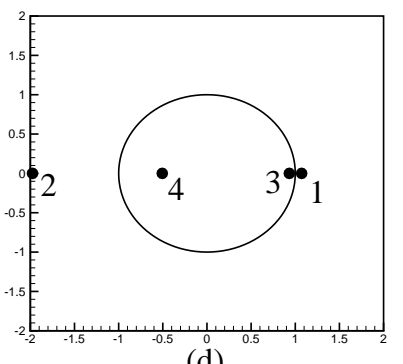

(d)

Figure 13. Eigenvalues of the mapping at a period for $\Omega=2, B o=100 . \epsilon=\mathrm{a}) 0.45 \mathrm{~b}) 0.93 \mathrm{c}$ ) 1.695 and d) 1.9275 . 


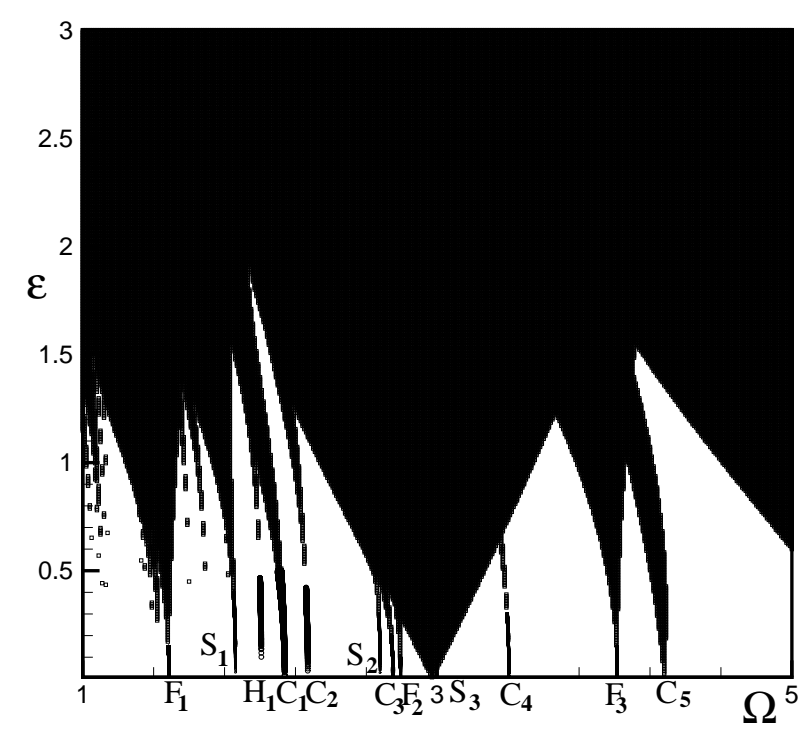

FiguRE 14. Stability plot, determined from the eigenvalues of the period mapping, using three modes. $B o=100$. The fundamental frequencies and major harmonics, subharmonics and combination resonances are marked.

frequency. Figure 16(b) shows the eigenvalues for $\epsilon \approx 0.3225$ corresponding to a point on the CRT emanating from $C_{1}$; two of the eigenvalues (the ones in the left half) have collided. Figure 16(c) shows the situation at a higher $\epsilon=1.3125$ corresponding to a point on the CRT emanating from $C_{2}$; the eigenvalues in the right half have just moved off the unit circle after collision. Finally, figure 16(d) shows a case where there is complete instability; we not only have a continuation of the earlier CRT from $C_{2}$ but also now instability due to the FRT issuing from subharmonic $S_{3}$ corresponding to the two real eigenvalues in the vicinity of -1 .

Finally, we show the behaviour of $a_{1}$ and $a_{2}$ for a point on the CRT shown in figure 10(c), for a $B o=100$ in figure 17. Since the CRT boundary demarcates stable and unstable regions, the real part of the Floquet exponent, $\mu_{r}=0$ for all points on its boundary. However, the imaginary part which controls the period is unknown and has to be determined. Recall that $\mu_{i}=0$ and 1 correspond to harmonic and subharmonic oscillations respectively. For a fixed drive frequency $\Omega$, we solve the eigenvalue problem with a trial value for $\mu_{i}$ between 0 and 1 and obtain the corresponding $\epsilon$. If this $\epsilon$ lies on the CRT boundary, that has already been determined from the period mapping, the trial value is the desired solution. If not, we change $\mu_{i}$ and iterate. The oscillations shown in figure 17 correspond to the temporal behaviour of $a_{1}$ and $a_{2}$ for the point $(2,0.70095)$ on a CRT boundary shown in figure 10(c). $\mu_{i}$ has been found to be approximately $0.602 i$ for this point. As can be expected, the oscillations, though stable are not periodic.

We now briefly discuss the relevance of these findings to experimental observation of Faraday waves. Faraday, as well as Rayleigh, observed only subharmonic oscillations while Matthiessen claimed to have seen harmonic ones and more than a century passed before Benjamin \& Ursell (1954) showed that both kinds of oscillations were possible. As shown by Muller et al (1997), the harmonic response is quite difficult to observe, 


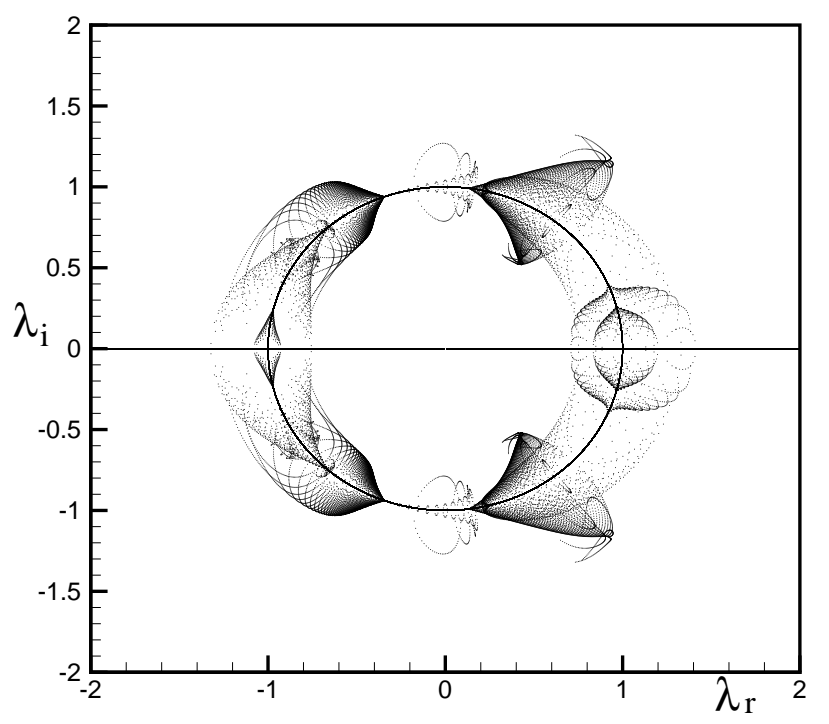

Figure 15. Eigenvalue plot, of the period mapping, using three modes. $B o=100$. The excursions off the unit circle indicate combination resonances. $\epsilon$ ranges from 0 to 3 .

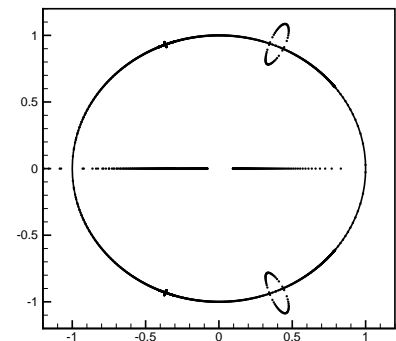

(a)

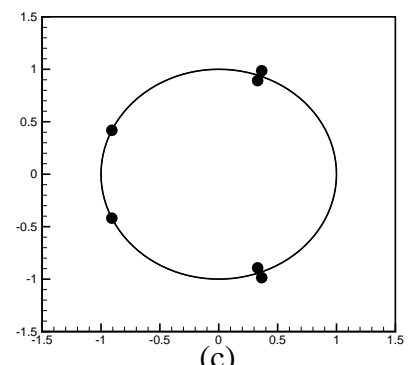

(c)

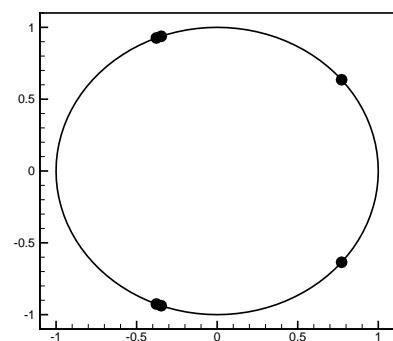

(b)

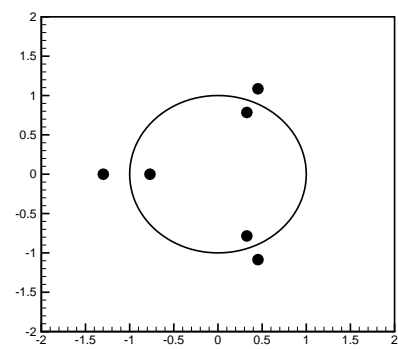

(d)

FigURE 16. Eigenvalues of the mapping at a period for $\Omega=2.12, B o=100 . N=3$ modes have been used. (a) $\epsilon$ ranges from 0 to $3 . \epsilon=$ b) 0.3225 c) 1.3125 and d) 1.44 . 


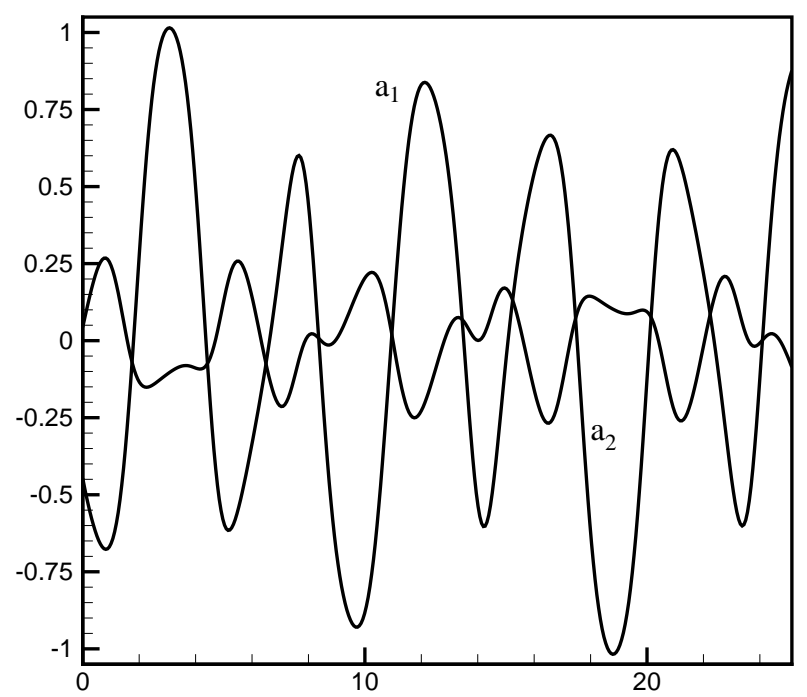

FiguRE 17. $a_{1}(t), a_{2}(t)$ for the point $(\Omega=2, \epsilon=0.70095)$ that lies on a CRT shown in figure 9 (c). $B o=100$ and the Floquet exponent $\mu=0.602 i$. The oscillations do not grow but are not periodic either.

occuring as it does at low drive frequencies. A few of the experiments have employed pinned contact lines though no responses other than harmonic / subharmonic have been reported for these cases. In future experiments, it will be interesting to look for almost periodic oscillations that occur on the boundaries of the CRTs. These would be more prominent for lower Bond numbers and one way to obtain these is by having smaller container dimensions. The occurrence of combination resonances makes it increasingly likely that neighbouring modes are in competition in larger parts of the $\Omega-\epsilon$ plane; it is known for example (Ciliberto \& Gollub 1984) that 'pattern competition takes place in regions close to the intersections of two stability curves' - the denser the tongues, the more such intersections, as in the present case, the more the competition and the more the possibility of different quasiperiodic and chaotic patterns emerging. Ciliberto \& Gollub (1984) model the evolution of each mode amplitude by a forced Mathieu equation which has, apart from damping and nonlinear terms (to fix the critical threshold and limit the mode growth respectively), also a gain coefficient in the forcing term; the interactions between different modes is sought to be described by coupling the gain coefficients. Thus, in effect, a system of coupled Mathieu equations is obtained. In the present case, the coupled system arises naturally as a consequence of the pinning of the contact line.

\section{Conclusion}

We have considered the effect of prescribing contact line conditions on inviscid Faraday waves in a circular cylinder. The present work extends the theoretical part of BU54 to the case of a pinned contact line. The main difference from the case of the freely moving contact line, considered for example in BU54, is that an infinite system of coupled Mathieu equations describes the situation in the present case; in the free case, they 
are uncoupled. A direct consequence is the appearance of new resonance tongues from the combination of the fundamental frequencies, which alone were present in the free contact line case. These interpenetrate the fundamental resonance tongues; the regions of instability increase as a result. Also, there is more interaction between nearby degenerate modes so that a variety of quasiperiodic and chaotic patterns can possibly emerge. On the boundaries of these tongues, almost periodic oscillations can occur. These have never been experimentally reported, to the best of our knowledge.

Since only an inviscid analysis was considered, the instability threshold touches the $x$ axis and damping estimates would have to be necessarily heuristic, as, for example in BU54. To obtain proper damping rates, the linearised NS equations have to be solved, as in Kumar \& Tuckerman (1994). Here, the contact line would have to be kept pinned to avoid the uncertainties associated with a moving contact line. However, effects of a moving contact line, in particular the calculation of the capillary damping, can be considered in an inviscid framework by including contact line conditions (for example, Hocking's (1987) or Miles' (1967) wetting conditions); Kidambi(2009a) demonstrates the production of capillary damping in this situation. The same mathematical framework can be used for both problems; the viscous problem with pinned contact line has been studied in Kidambi (2009b). Another interesting problem is that of polychromatic excitation keeping the contact line still pinned. These are being pursued and will be reported elsewhere.

This work is funded by the CSIR Supra Institutional Project SIP-CFD-08.

\section{REFERENCES}

Bechhoefer, J. , Ego, V. , Manneville, S. \& Johnson, B. 1995 An experimental study of the onset of parametrically pumped surface waves in viscous fluids. J. Fluid Mech. 288, 325 350 .

Benjamin, T. B. \& Ursell, F. 1954 The stability of the plane free surface of a liquid in vertical periodic motion. Proc. Roy. Soc. Lond. A 225(1163), $505-515$.

Beyer, J. \& Friedrich, R. 1995 Faraday instability : linear analysis for viscous fluids. Phys. Rev. E 51(2), 1162 - 1168.

Bhattacherjee, A. B. 2008 Faraday instability in a two-component Bose Einstein condensate. Phys. Scr. 78(4), 045009.

Cerda, E. A. \& Tirapegui, E. L. 1998 Faraday's instability in viscous fluid. J. Fluid Mech. 368, $195-228$.

Christiansen, B. , Alstrom, P. \& Levinsen M. T. 1995 Dissipation and ordering in capillary waves at high aspect ratios. J. Fluid Mech. 291, 323 - 341.

Ciliberto, S. \& Gollub, J. P. 1984 Pattern competition leads to chaos. Phys. Rev. Lett. 52(11), $922-925$.

Douady, S. 1990 Experimental study of the Faraday instability. J. Fluid Mech. 221, 383 - 409.

Edwards, W. S. \& Fauve, S. 1993 Parametrically excited quasicrystalline waves. Phys. Rev. E 47(2), R778 - R791.

Edwards, W. S. \& Fauve, S. 1994 Patterns and quasi-patterns in the Faraday experiment. J. Fluid Mech. 278, 123 - 148.

Hansen, J. 1985 Stability diagrams of coupled Mathieu equations, Ingenieur-Archiv 55, 463 473.

Hill, G. W. 1886 On the part of the motion of lunar perigee which is function of the mean motions of the sun and moon. Acta Math. 8, $1-36$.

Hocking, L. M. 1987 The damping of capillary-gravity waves at a rigid boundary. J. Fluid Mech. $179,253-266$.

Kidambi, R. \& Shankar, P. N. 2004 The effects of the contact angle on sloshing in containers. Proc. R. Soc. Lond. A 460, $2251-2267$.

Kidambi, R. 2009a Capillary damping of inviscid surface waves in a circular cylinder. J. Fluid Mech. 627, 323 - 340. 
Kidambi, R. 2009b Meniscus effects on the frequency and damping of capillary-gravity waves in a brimful circular cylinder. Wave Motion 46, 144 - 154.

Kumar, K. \& Tuckerman, L. S. 1994 Parametric instability of the interface between two fluids. J. Fluid Mech. 279, 49 - 68.

Kumar, K. 1996 Linear theory of Faraday instability in viscous liquids. Proc. R. Soc. Lond. A $452,1113-1126$.

Lam, K. D. N. T. \& Caps, H. 2011 Effect of a capillary meniscus on the Faraday instability threshold. Eur. Phys. J. E 34, 112.

Lindh, K. G. \& Likins, P. W. 1970 Infinite determinant methods for stability analysis of periodiccoefficient differential equations. AIAA Jl. V 8, No. 4.

Mathieu, E. 1868 Le mouvement vibratoire d'une membrane de forme elliptique. J. Math. Pures Appl. 13, 137 - 203.

McLachlan, G. 1947 Theory and application of Mathieu functions. Oxford University Press.

Miles, J. W. 1967 Surface-wave damping in closed basins. Proc. Roy. Soc. Lond. A 297, 459 475.

Milner, S. T. 1991 Square patterns and secondary instabilities in driven capillary waves. $J$. Fluid Mech. 225, 81 - 100.

Muller, H. W. Wittmer, H. , Wagner, C. , Albers, J. \& Knorr, K. 1997 Analytic stability theory for Faraday waves and the observation of the harmonic surface response. Phys. Rev. Lett. 78(12), 2357 - 2360.

Nayfeh, A. H. \& Mook, D. T. 1995 Nonlinear oscillations. John Wiley $\&$ Sons.

Ozakin, A. \& Shaikh, F. 2011 Stability analysis of surface ion traps. arXiv : 1109.2160v

Rayleigh, Lord 1883 On the crispation of fluid resting upon a vibrating support. Phil. Mag. $15(5), 50-58$. 